\title{
Diabetes-related distress and its associated factors among patients with type 2 diabetes
}

mellitus in China

Huanhuan Zhou ${ }^{\mathrm{a}, \mathrm{b}, \mathrm{c}}$, Junya Zhu ${ }^{\mathrm{d}}$, Lin Liu ${ }^{\mathrm{a}, \mathrm{b}}$, Fan Lia ${ }^{\mathrm{a}, \mathrm{b}}$, Anne F. Fish ${ }^{\mathrm{e}}$, Tao Chen ${ }^{\mathrm{f}}$, Qingqing $\operatorname{Lou}^{\mathrm{a}, \mathrm{b}, *}$

aAffiliated Hospital of Integrated Traditional Chinese and Western Medicine, Nanjing University of Chinese Medicine, Nanjing, Jiangsu Province, China.

bJiangsu Province Academy of Traditional Chinese Medicine, Nanjing, Jiangsu Province, China.

'Nursing College, Nanjing University of Chinese Medicine, Nanjing, Jiangsu Province, China.

dDepartment of Health Policy and Management Johns Hopkins Bloomberg School of Public Health 624 North Broadway Baltimore, MD, USA.

${ }^{e}$ College of Nursing (ISP Program), University of Missouri-St. Louis, St. Louis, MO, USA.

fDepartment of Clinical Sciences Liverpool School of Tropical Medicine Pembroke Place, Liverpool L3 5QA, UK.

* Corresponding Author: Tel: +8615312019129 ; Fax: +02585502829

E-mail adress: 1qq188@yahoo.com (Q.Q. Lou) 


\begin{abstract}
Diabetes-related distress is one of the psychological disorders affecting patients with diabetes, yet there are few studies about diabetes-related distress in Chinese patients. To assess the level of psychological distress and examine its associated factors, we conducted a cross-sectional analysis of patients with type 2 diabetes mellitus from a Chinese tertiary hospital. The Diabetes Distress Scale (DDS) and the General Self-Efficacy Scale (GSES) were administered. There were $210(57.85 \%)$ patients with little or no diabetes-related distress, $84(23.14 \%)$ with moderate diabetes-related distress and 69 (19.01\%) with high diabetes-related distress. Stepwise multiple linear regression showed that sleep time was significantly related to the DDS total score and the subscale scores of emotional burden (EB) ( $\beta=-0.190,-0.379)$, respectively. GSES was associated with the DDS total score $(\beta=-0.128)$ and the EB score $(\beta=-0.153)$. Oral medication plus insulin was significantly related to regimen-related distress $(\mathrm{RD})(\beta=0.137)$, physician-related distress $(\mathrm{PD})(\beta=0.152)$ and interpersonal distress (ID) $(\beta=0.103)$. Physical activity $(\beta=-0.185)$ and making meal plan with health care professionals(HCP) $(\beta=-0.169)$ were associated with $\mathrm{RD}$. The prevalence of diabetes-related distress among patients with type 2 diabetes mellitus was high in China. DDS and EB were associated with poorer sleep time and lower self-efficacy. Interventions to improve sleep are needed. Qualitative and longitudinal studies are required to understand why type 2 diabetic patients are not getting enough sleep.
\end{abstract}

Key words Chinese; Diabetes; Diabetes-related distress; Self-efficacy; Sleep time, Insulin, Physical activity 


\section{Introduction}

Living with diabetes can be challenging and stressful. Research has shown that anxiety disorders and depression are the most common psychological comorbid conditions among patients with type 2 diabetes mellitus (Kawada 2016; Brieler et al., 2016). Particularly, the prevalence of depression among this group of patients is 1.5-3.0 times higher than that found in the general population (Munhoz et al., 2015; Park and Brown, 2015). Depression has been shown to adversely affect diabetes treatment outcomes and patient self-management behaviors. Diabetes-related distress includes negative emotional reactions to the diagnosis, the threat of complications and self-management demands that add much stress to patients' day to day living (Stanković et al., 2013). Diabetes-related distress, depression, and subclinical depression are all psychologic disorders affecting patients with diabetes (Chew et al., 2016; Zhang et al., 2013). Zhang et al. (2013) found that diabetes-related distress is a predictor of depression and plays an important role in treatment adherence. Therefore, screening for diabetes-related distress is important for primary prevention of depression and other psychologic problems in diabetes patients. There is evidence to suggest that poor sleep was related to a decline in the living quality of patients with diabetes (Luyster and Dunbar-Jacob, 2011). The relationship between depression and sleep has been widely studied in China (Zhao and Li, 2016; Zhang and Lou, 2016), yet there is no research about the relationship between sleep time and diabetes-related distress in China.

Maintaining an appropriate glycemic control is important to prevent complications of diabetes. The American Diabetes Association guidelines (American Diabetes Association, 2016) recommend that a reasonable HbAlc goal for type 2 diabetes mellitus patients is < 7\%, but many people do not meet the treatment goal (Ali et al., 2013). Emotional distress made the required self-management of the disease more difficult and limited the patients' management of self-care activities necessary to achieve an adequate glycemic control (Aikens, 2012). In both cross-sectional and longitudinal analyses, Fisher et al. (2010) found that diabetes-related distress, but not clinical depression or depressive symptoms, is associated with HbA1c. Moreover, in patients with diabetes, the prevalence of distress is much higher than depression (Nicolucci et al., 2013), so it is crucial to evaluate the 
relationship between diabetes-related distress and HbAlc among patients with type 2 diabetes mellitus in China. Apart from HbA1c, other related factors such as age, gender, treatment adherence and social support have been examined (Polonsky et al., 2005; Ogbera and Adeyemi-Doro, 2011; Karlsen and Bru,2014) in Caucasian, we found one study analyzed the relationship between diabetes-related distress and its related factors in China(Kong et al., 2013), but the subjects were type 1 diabetes patients. Therefore, the aims of the current study were to examine the prevalence of diabetes-related distress and evaluate its associated factors in patients with type 2 diabetes mellitus in China.

\section{Methods}

\subsection{Design and participants}

This cross-sectional survey was conducted at a tertiary hospital in Nanjing, Jiangsu Province, China using a convenience sample of patients with type 2 diabetes mellitus who visited the hospital between June and December 2014. The inclusion criteria were patients aged 18 years or older, had type 2 diabetes mellitus for at least 3 months and were able to communicate fluently and clearly. Exclusion criteria were pregnancy, type 1 diabetes, dementia, psychosis, or severe illnesses such as cancer. Patients who met the eligibility criteria and signed the informed consent were recruited to complete the surveys. This study was approved by the hospital's Ethics Committee. The five nurse researchers trained as data collectors used uniform instructions in order to guarantee the quality of data collection. Patients' demographic and clinical characteristics were collected from the medical records. The data collectors obtained further information face to face on sleep time, hypoglycemia and complications. All questionnaires were completed by patients in a quiet room with the assistance of a data collector. The same language guide was used when patients were completing the surveys.

Three hundred and sixty-three out of 384 eligible patients completed the questionnaires, representing a $94.5 \%$ response rate. Their ages ranged from 23 to 82 years of age. Age was divided into three groups according to age criteria for the classification of the World Health Organization. Mean age of the youth, middle-aged and elderly were $36.6 \pm 6.3 \mathrm{y}, 52.4 \pm 4.1 \mathrm{y}$, and $66.2 \pm 4.9 \mathrm{y}$. 


\subsection{Measurements}

\subsubsection{Chinese version of Diabetes Distress Scale (DDS)}

The DDS was developed by Polonsky et al. (2005) to evaluate psychological distress of patients with diabetes. The scale has established reliability (Cronbach's alpha of 0.88-0.93). In 2010, Yang et al. (2010) translated the scale into Chinese, and reported Cronbach's alphas of 0.84-0.95 and test-retest reliability of 0.849 in Chinese patients. The Chinese DDS includes 17 items measuring four dimensions: emotional burden (EB, 5 items), physician-related distress ( $\mathrm{PD}, 4$ items), regimen-related distress (RD, 5 items), and diabetes-related interpersonal distress (ID, 3 items). These items use a six-point Likert scale ranging from 1 (no distress) to 6 (high distress). A total score was calculated by adding the 17 items. Higher scores indicate greater distress (Graue et al., 2012). According to the revised rating system developed by Fisher et al. (2012), a mean item score $<2$ indicates little or no distress; 2-3 indicates moderate distress; and $>3$ indicates high distress.

\subsubsection{Chinese version of General Self-Efficacy Scale (GSES)}

The GSES, developed by Schwarzer et al. (1997), was used to evaluate patients' self-efficacy. After modification, the final version includes 10 items from the original 20 items. Zhang et al. (1995) translated the scale into Chinese. Each item contains 4 options: 1 (totally wrong), 2 (basically right), 3 (almost right) and 4 (absolutely right). The internal consistency reliability was 0.87 , the test-retest reliability was 0.83 and the correlation coefficient among the total score and each item ranged from $0.60-0.77$ (Zhang and Schwarzer, 1995).

\subsubsection{Calculation of sleep time}

The majority of Chinese people have a habit of taking a daily noon time nap, and nap is an important part of daily sleep for Chinese people. In our study, the sleep time included night sleep time and nap time $(30 \sim 120$ mins). When we analyze the relationship between sleep time and diabetes-related distress and the dimensions of diabetes-related distress, we use the total sleep time (night sleep + nap). 


\subsection{Statistical methods}

SPSS version 15.0 (SPSS Inc., Chicago, IL, USA) was used to carry out statistical analyses. Values were reported as mean $\pm \mathrm{SD}$ or frequency and percent where applicable. We used Shapiro-Wilk test to examine the distribution of the measurement data. The independent two-sample T-test and analysis of variance (ANOVA) for multiple independent samples were performed for the data with a normal distribution. Non-parametric tests including Mann-Whitney $U$ test and Kruskal-Wallis $H$ test were used for data that did not exhibit a normal distribution. Spearman correlation was used to examine relationship between diabetes-related distress (with four subscales) and patient characteristics, which including diabetes duration, weight, BMI, times of diabetes education, physical activity, exercise time, making diet plan with medical staff, treatment regimen, lipid profile, SBP, DBP and HbA1c and GSES. A stepwise multiple regression model was performed. DDS and four subscales were the dependent variables. Independent variables were those that statistically correlated to diabetes-related distress. If the $\alpha \leq 0.05$, the variables were entered into the model, and the variables were ruled out with $\alpha \geq 0.10$. A two-tailed $\alpha=0.05$ was considered significant. Standardized coefficients $\beta$ were used to directly reflect to what extent the independent variables affect the dependent variables.

\section{Results}

\subsection{DDS total scores in patients' overall features and 3 columns with patients' characteristics stratified according to the different diabetes-related distress categories}

Demographic and clinical characteristics included Age $(55.3 \pm 11.7 \mathrm{y})$, Male $(58.1 \%)$, Female $(41.9 \%)$, HbA1c $(9.7 \pm 2.6 \%)$, weight $(67.2 \pm 11.8 \mathrm{~kg}), \mathrm{BMI}\left(24.5 \pm 3.3 \mathrm{~kg} / \mathrm{m}^{2}\right), \mathrm{SBP}$ $(129.7 \pm 16.1 \mathrm{mmHg})$, DBP $(80.6 \pm 9.8 \mathrm{mmHg})$, Triglycerides $(40.3 \pm 34.9 \mathrm{mg} / \mathrm{dl})$, Total cholesterol $(44.5 \pm 27.9 \mathrm{mg} / \mathrm{dl})$, Low-density lipoprotein cholesterol $(53.3 \pm 25.4 \mathrm{mg} / \mathrm{dl})$, High-density lipoprotein cholesterol $(21.4 \pm 13.1 \mathrm{mg} / \mathrm{dl})$ and GSES $(2.8 \pm 1.0)$, duration of diabetes $(7.1 \pm 6.3 y)$ and GSES $(2.8 \pm 1.0)$ for the total sample and also stratified according to the different diabetes-related distress categories are reported in Table 1. We compared the 
DDS total scores across groups of patients with different characteristics. Married patients had a lower score than those who were unmarried, widowed or divorced $(\mathrm{P}=0.033)$. Patients who did not use any medication to control blood glucose got the lowest scores, while those who used oral plus insulin got the highest scores $(\mathrm{P}=0.047)$. And inactive people got higher score $(\mathrm{P}=0.029)$ 
Table 1

DDS total scores in patients' overall features and 3 columns with patients' characteristics stratified according to the different diabetes-related distress categories $(n=363)$

\begin{tabular}{|c|c|c|c|c|c|c|c|c|c|c|c|}
\hline Variable & $\begin{array}{l}\text { Number } \\
(\%)\end{array}$ & $\begin{array}{l}\text { DDS } \\
\text { total } \\
\text { score }\end{array}$ & $\mathrm{F}$ & df & $\begin{array}{l}\mathrm{P} \\
\text { value }\end{array}$ & $\begin{array}{l}\text { Little or no } \\
\text { diabetes-related } \\
\text { distress } \\
\text { Number (\%) }\end{array}$ & $\begin{array}{l}\text { Moderate } \\
\text { diabetes-related } \\
\text { distress } \\
\text { Number (\%) }\end{array}$ & $\begin{array}{l}\text { High } \\
\text { diabetes-related } \\
\text { distress } \\
\text { Number (\%) }\end{array}$ & $\mathrm{X}^{2}$ & $\mathrm{df}$ & $\begin{array}{l}\mathrm{P}^{*} \\
\text { value }\end{array}$ \\
\hline Total & 363 & $\begin{array}{l}32.27 \\
(13.09)\end{array}$ & & & & $210(57.9)$ & $84(23.1)$ & $69(19.0)$ & & & \\
\hline Gender & & & 0.47 & 1 & 0.495 & & & & 6.393 & 2 & 0.041 \\
\hline Male (\%) & $\begin{array}{l}211 \\
(58.1)\end{array}$ & $\begin{array}{l}32.67 \\
(12.85)\end{array}$ & & & & $115(54.5)$ & $57(27.0)$ & $39(18.5)$ & & & \\
\hline Female $(\%)$ & $\begin{array}{l}152 \\
(41.9)\end{array}$ & $\begin{array}{l}31.72 \\
(13.44)\end{array}$ & & & & $95(62.5)$ & $27(17.8)$ & 30 (19.7) & & & \\
\hline Age (year) & $\begin{array}{l}55.3 \\
(11.7)\end{array}$ & & 2.27 & 2 & 0.316 & & & & 10.781 & 4 & 0.029 \\
\hline $18-44(\%)$ & $64(17.6)$ & $\begin{array}{l}34.47 \\
(15.24)\end{array}$ & & & & $32(50.0)$ & $18(28.1)$ & $14(0.218)$ & & & \\
\hline $45-59(\%)$ & $\begin{array}{l}156 \\
(43.0)\end{array}$ & $\begin{array}{l}30.70 \\
(10.67)\end{array}$ & & & & $92(59.0)$ & $40(25.6)$ & $24(15.4)$ & & & \\
\hline$\geq 60(\%)$ & $\begin{array}{l}143 \\
(39.4)\end{array}$ & $\begin{array}{l}33.00 \\
(14.29)\end{array}$ & & & & $86(60.1)$ & $26(18.2)$ & $31(21.7)$ & & & \\
\hline $\begin{array}{c}\text { Educational level } \\
\text { Illiteracy (\%) }\end{array}$ & $35(9.6)$ & $\begin{array}{l}30.20 \\
(9.22)\end{array}$ & 0.86 & 4 & 0.492 & $23(65.7)$ & $6(17.1)$ & $6(17.1)$ & 9.439 & 6 & 0.150 \\
\hline
\end{tabular}




\begin{tabular}{|c|c|c|c|c|c|c|c|c|c|c|c|}
\hline Primary school (\%) & $57(15.7)$ & $\begin{array}{l}34.00 \\
(16.34)\end{array}$ & & & & $31(54.4)$ & $12(21.1)$ & $14(24.6)$ & & & \\
\hline Middle school (\%) & $\begin{array}{l}106 \\
(29.2)\end{array}$ & $\begin{array}{l}33.36 \\
(12.91)\end{array}$ & & & & $57(53.8)$ & $27(25.5)$ & $22(20.8)$ & & & \\
\hline $\begin{array}{l}\text { Senior } \\
\text { High/Polytechnic school } \\
(\%)\end{array}$ & $\begin{array}{l}103 \\
(28.4)\end{array}$ & $\begin{array}{l}31.16 \\
(13.03)\end{array}$ & & & & $63(61.2)$ & $26(25.2)$ & $14(13.6)$ & & & \\
\hline $\begin{array}{l}\text { Bachelor degree or } \\
\text { above }(\%)\end{array}$ & $62(17.6)$ & $\begin{array}{l}31.84 \\
(12.01)\end{array}$ & & & & $36(58.1)$ & $13(21.0)$ & $13(20.9)$ & & & \\
\hline Marital status & & & 0.85 & 1 & 0.033 & & & & 13.642 & 2 & 0.001 \\
\hline Married (\%) & $\begin{array}{l}344 \\
(94.8)\end{array}$ & $\begin{array}{l}31.86 \\
(12.70)\end{array}$ & & & & $203(59.0)$ & $81(23.5)$ & $60(17.5)$ & & & \\
\hline $\begin{array}{l}\text { Unmarried, widowed, } \\
\text { divorced }(\%)\end{array}$ & $19(5.2)$ & $\begin{array}{l}39 \\
(17.57)\end{array}$ & & & & $7(36.8)$ & $3(15.8)$ & $9(47.4)$ & & & \\
\hline Diabetes Duration (year) & $7.1(6.3)$ & & 2.95 & 3 & 0.544 & & & & 22.173 & 6 & 0.001 \\
\hline$<5(\%)$ & $\begin{array}{l}155 \\
(42.7)\end{array}$ & $\begin{array}{l}31.16 \\
(11.62)\end{array}$ & & & & $85(54.8)$ & $45(29.0)$ & $25(16.1)$ & & & \\
\hline $5-10(\%)$ & $91(25.1)$ & $\begin{array}{l}33.49 \\
(13.64)\end{array}$ & & & & $50(54.9)$ & $20(22.0)$ & $21(23.1)$ & & & \\
\hline $10-15(\%)$ & $75(20.7)$ & $\begin{array}{l}33.00 \\
(16.00)\end{array}$ & & & & $52(69.3)$ & $9(12.0)$ & $14(18.7)$ & & & \\
\hline$>15(\%)$ & $42(11.6)$ & $\begin{array}{l}32.40 \\
(11.27)\end{array}$ & & & & $23(54.8)$ & $10(23.8)$ & $9(21.4)$ & & & \\
\hline Treatment regimen & & & 1.68 & 3 & 0.047 & & & & 7.187 & 4 & 0.126 \\
\hline $\begin{array}{l}\text { Non-hypoglycemic } \\
\text { agents }(\%)\end{array}$ & $32(8.8)$ & $\begin{array}{l}28.86 \\
(8.85)\end{array}$ & & & & $21(65.6)$ & $7(21.9)$ & $4(12.5)$ & & & \\
\hline Oral hypoglycemic & $96(26.5)$ & 31.13 & & & & $61(63.5)$ & $21(21.9)$ & $14(14.6)$ & & & \\
\hline
\end{tabular}




\begin{tabular}{|c|c|c|c|c|c|c|c|c|c|c|c|}
\hline agents $(\%)$ & & $(13.83)$ & & & & & & & & & \\
\hline Insulin (\%) & $89(24.5)$ & $\begin{array}{l}32.39 \\
(12.43)\end{array}$ & & & & $49(55.1)$ & $24(27.0)$ & $16(17.9)$ & & & \\
\hline Oral plus Insulin (\%) & $\begin{array}{l}146 \\
(40.2)\end{array}$ & $\begin{array}{l}33.74 \\
(13.64)\end{array}$ & & & & $79(54.1)$ & $32(21.9)$ & $35(24.0)$ & & & \\
\hline Diabetes education & & & 0.90 & 1 & 0.495 & & & & 0.575 & 2 & 0.750 \\
\hline Yes (\%) & $\begin{array}{l}101 \\
(27.8)\end{array}$ & $\begin{array}{l}32.29 \\
(12.42)\end{array}$ & & & & $59(58.4)$ & $21(20.8)$ & $21(20.8)$ & & & \\
\hline No $(\%)$ & $\begin{array}{l}262 \\
(72.2)\end{array}$ & $\begin{array}{l}32.26 \\
(13.36)\end{array}$ & & & & $151(57.6)$ & $63(24.0)$ & $48(18.3)$ & & & \\
\hline Physical activity & & & 4.79 & 1 & 0.029 & & & & 2.814 & 2 & 0.245 \\
\hline Yes $(\%)$ & $\begin{array}{l}218 \\
(60.1)\end{array}$ & $\begin{array}{l}31.05 \\
(12.10)\end{array}$ & & & & $126(57.8)$ & $49(2.3)$ & $26(11.9)$ & & & \\
\hline No $(\%)$ & $\begin{array}{l}145 \\
(39.9)\end{array}$ & $\begin{array}{l}34.10 \\
(14.30)\end{array}$ & & & & $84(57.9)$ & $35(24.1)$ & $26(17.9)$ & & & \\
\hline $\begin{array}{l}\text { Whether meal plan had } \\
\text { been developed by HCP } \\
\text { and the patient }\end{array}$ & & & 3.20 & 1 & 0.113 & & & & 3.040 & 2 & 0.219 \\
\hline Yes $(\%)$ & $92(25.3)$ & $\begin{array}{l}30.17 \\
(12.26)\end{array}$ & & & & $57(62.0)$ & $18(19.6)$ & $17(18.4)$ & & & \\
\hline No (\%) & $\begin{array}{l}271 \\
(74.7)\end{array}$ & $\begin{array}{l}32.98 \\
(13.31)\end{array}$ & & & & $153(56.4)$ & $66(24.4)$ & $52(19.2)$ & & & \\
\hline Complications & & & & & & & & & & & \\
\hline $\begin{array}{l}\text { diabetes retinopathy } \\
(\%)\end{array}$ & $15(4.1)$ & $\begin{array}{l}32.9 \\
(13.24)\end{array}$ & 0.04 & 1 & 0.841 & $11(73.3)$ & $1(6.7)$ & $3(20.0)$ & 2.054 & 2 & 0.358 \\
\hline $\begin{array}{l}\text { diabetes nephropathy } \\
(\%)\end{array}$ & $9(2.5)$ & $\begin{array}{l}38.00 \\
(15.94)\end{array}$ & 1.77 & 1 & 0.184 & $7(77.8)$ & $2(22.2)$ & $0(0)$ & 2.048 & 2 & 0.359 \\
\hline diabetes neuropathy & $9(2.5)$ & 39.44 & 2.79 & 1 & 0.096 & $5(55.6)$ & $3(33.3)$ & $1(11.1)$ & 3.043 & 2 & 0.220 \\
\hline
\end{tabular}




\begin{tabular}{|c|c|c|c|c|c|c|c|c|c|c|c|}
\hline$(\%)$ & & $(13.96)$ & & & & & & & & & \\
\hline diabetic foot $(\%)$ & $3(0.83)$ & $\begin{array}{l}24.50 \\
(10.61)\end{array}$ & 0.71 & 1 & 0.401 & $2(33.7)$ & $0(0)$ & $1(33.3)$ & 1.031 & 2 & 0.597 \\
\hline Whether & 363 & & 1.66 & 1 & 0.265 & & & & 2.716 & 2 & 0.257 \\
\hline \multicolumn{12}{|l|}{ hypoglycemia or not (\%) } \\
\hline Yes (\%) & $152(41.9)$ & $\begin{array}{l}33.22 \\
(13.92)\end{array}$ & & & & $91(59.9)$ & $30(19.7)$ & $23(15.1)$ & & & \\
\hline No (\%) & $\begin{array}{l}211 \\
(58.1)\end{array}$ & $\begin{array}{l}31.65 \\
(12.51)\end{array}$ & & & & $119(56.4)$ & $54(25.6)$ & $46(21.8)$ & & & \\
\hline
\end{tabular}

$\mathrm{P}$ value, comparisons of total scores in patients' overall features i.e. gender, age, and education level and so on.

Variables are expressed as the mean \pm standard deviation, or percent.

GSES, General Self-Efficacy Scale; HCP, health care professional. 


\subsection{DDS scores and the distribution of diabetes-related distress in patients}

The average total score of DDS was $32.3 \pm 13.1$, and each subscale score was between 4.7 to 11.6. In four subscales, RD got the highest score, and ID was the lowest. A total of 210 (57.6\%) patients had little or no diabetes-related distress, 84 (23.1\%) had moderate diabetes-related distress, and 69 (19.0\%) had high diabetes-related distress. (See Table 2) Table 2

The scores of DDS and subscales $\left(\bar{X}_{ \pm}\right.$s) and the distribution of diabetes-related distress levels (\%)

\begin{tabular}{|c|c|c|c|c|c|c|c|c|c|c|}
\hline \multirow[t]{2}{*}{ Scale } & \multirow[t]{2}{*}{$\begin{array}{l}\text { Number } \\
\text { of Items }\end{array}$} & \multirow[t]{2}{*}{$\begin{array}{l}\text { Total } \\
\text { Score } \\
\left(\bar{X}_{ \pm \mathrm{s}}\right)\end{array}$} & \multirow[t]{2}{*}{$\begin{array}{l}\text { Mean } \\
\text { Score }\end{array}$} & \multicolumn{2}{|c|}{$\begin{array}{l}\text { Little or no } \\
\text { diabetes-related } \\
\text { distress }\end{array}$} & \multicolumn{2}{|c|}{$\begin{array}{l}\text { Moderate } \\
\text { diabetes-related } \\
\text { distress }\end{array}$} & \multicolumn{2}{|c|}{$\begin{array}{l}\text { High } \\
\text { diabetes-related } \\
\text { distress } \\
\end{array}$} & \multirow[t]{2}{*}{$P$ value } \\
\hline & & & & $\mathrm{N}(\%)$ & MS & $\mathrm{N}(\%)$ & MS & N (\%) & MS & \\
\hline $\begin{array}{c}\text { DDS } \\
\text { (total scores) }\end{array}$ & 17 & $\begin{array}{l}32.3 \\
(13.1)\end{array}$ & 1.9 & $\begin{array}{l}210 \\
(57.9)\end{array}$ & $\begin{array}{l}1.4 \\
(0.2)\end{array}$ & $\begin{array}{l}84 \\
(23.1)\end{array}$ & $\begin{array}{l}2.2 \\
(0.2)\end{array}$ & $\begin{array}{l}69 \\
(19.0)\end{array}$ & $\begin{array}{l}3.2 \\
(0.7)\end{array}$ & \\
\hline EB & 5 & $\begin{array}{l}10.3 \\
(5.2)\end{array}$ & 2.1 & $\begin{array}{l}301 \\
(82.9)\end{array}$ & $\begin{array}{l}1.4 \\
(0.3)\end{array}$ & $\begin{array}{l}35 \\
(9.6)\end{array}$ & $\begin{array}{l}2.3 \\
(0.3)\end{array}$ & $\begin{array}{l}27 \\
(7.4)\end{array}$ & $\begin{array}{l}4.0 \\
(0.7)\end{array}$ & $<0.001$ \\
\hline PD & 4 & $\begin{array}{l}5.7 \\
(3.5)\end{array}$ & 1.4 & $\begin{array}{l}166 \\
(45.7)\end{array}$ & $\begin{array}{l}1.1 \\
(0.2)\end{array}$ & $\begin{array}{l}96 \\
(26.5)\end{array}$ & $\begin{array}{l}2.4 \\
(0.4)\end{array}$ & $\begin{array}{l}101 \\
(27.8)\end{array}$ & $\begin{array}{l}4.4 \\
(1.0)\end{array}$ & $<0.001$ \\
\hline $\mathrm{RD}$ & 5 & $\begin{array}{l}11.6 \\
(5.6)\end{array}$ & 2.3 & $\begin{array}{l}261 \\
(71.9)\end{array}$ & $\begin{array}{l}1.4 \\
(0.3)\end{array}$ & $\begin{array}{l}62 \\
(17.1)\end{array}$ & $\begin{array}{l}2.4 \\
(0.4)\end{array}$ & $\begin{array}{l}40 \\
(11.0)\end{array}$ & $\begin{array}{l}4.0 \\
(0.7)\end{array}$ & $<0.001$ \\
\hline ID & 3 & $\begin{array}{l}4.7 \\
(2.7)\end{array}$ & 1.6 & $\begin{array}{l}233 \\
(64.2) \\
\end{array}$ & $\begin{array}{l}1.1 \\
(0.2)\end{array}$ & $\begin{array}{l}99 \\
(27.3)\end{array}$ & $\begin{array}{l}2.4 \\
(0.4)\end{array}$ & $\begin{array}{l}31 \\
(8.5)\end{array}$ & $\begin{array}{l}4.1 \\
(0.7)\end{array}$ & $<0.001$ \\
\hline
\end{tabular}

Scores are expressed as the mean \pm standard deviation, or percent.

$\mathrm{EB}$, emotional burden subscale; $\mathrm{PD}$, physician-related distress subscale; RD, regimen-related distress subscale; ID, diabetes-related interpersonal distress subscale; N, number; MS, mean score.

\subsection{Spearman correlation}

Diabetes duration, weight, BMI, sleep time, times of diabetes education, physical activity, exercise time, whether meal plan had been developed by HCP and the patient, treatment regimen, TG, SBP, and HbA1c and GSES were significantly related to diabetes-related distress. The results are shown in Table 3.

Table 3

Spearman Correlation between diabetes-related distress and related factors 


\begin{tabular}{llllll}
\hline Valuable & $\begin{array}{l}\text { Total } \\
\text { Score of }\end{array}$ & & PB & RD & ID \\
& DDS & & & & \\
\hline Diabetes duration & 0.048 & 0.031 & -0.036 & $0.117^{*}$ & -0.049 \\
Weight & -0.056 & $-0.144^{* *}$ & 0.042 & -0.032 & -0.029 \\
BMI & 0.882 & $-0.172^{* *}$ & -0.018 & -0.030 & -0.067 \\
Sleep time & $-0.148^{* *}$ & $-0.165^{* *}$ & 0.009 & $-0.137^{*}$ & -0.072 \\
Times of Diabetes education & 0.105 & $0.285^{*}$ & 0.007 & 0.094 & -0.036 \\
Physical activity & -0.106 & -0.012 & 0.029 & $-0.150^{* *}$ & -0.083 \\
Exercise time & $-0.158^{*}$ & $-0.165^{*}$ & -0.053 & -0.111 & -0.016 \\
Whether meal plan had been & $-0.119^{*}$ & -0.017 & -0.070 & $-0.142^{*}$ & 0.011 \\
developed by HCP and the patient & & & & & \\
Treatment regimen & $0.138^{* *}$ & 0.008 & 0.077 & $0.127^{*}$ & 0.065 \\
TG & $-0.106^{*}$ & -0.070 & 0.001 & -0.085 & -0.044 \\
TC & 0.029 & -0.031 & 0.038 & 0.015 & -0.024 \\
HDL-C & 0.028 & 0.071 & -0.027 & 0.017 & 0.030 \\
LDL-C & -0.028 & -0.072 & 0.009 & -0.044 & -0.040 \\
SBP & 0.044 & -0.041 & $0.117^{*}$ & 0.026 & 0.048 \\
DBP & -0.018 & -0.058 & 0.016 & -0.016 & -0.003 \\
HbA & $0.102^{*}$ & 0.023 & 0.085 & $0.103^{*}$ & 0.012 \\
GSES & $-0.167^{* *}$ & $-0.186^{* *}$ & -0.098 & $-0.113^{*}$ & $-0.136^{*}$ \\
\hline
\end{tabular}

$* \mathrm{P}<0.05, * * \mathrm{P}<0.01$

BMI, body mass index; DBP, diastolic blood pressure; SBP, systolic blood pressure; TG, triglycerides; TC, total cholesterol; HDL-C, high-density lipoprotein cholesterol; LDL-C, low-density lipoprotein cholesterol; HCP, health care professional.

\subsection{Stepwise multiple linear regression}

We next examined the DDS total score in relation to seven statistically significant variables, including total daily sleep time, physical activity $(\mathrm{no}=0$, yes $=1)$, whether the meal plan was developed by HCP and the patient (no=0, yes=1), treatment regimen, TG, HbA1c and GSES. Stepwise multiple linear regression showed that sleep time $(\beta=-0.190, \mathrm{P}=0.029)$ and GSES $(\beta=-0.128, \mathrm{P}=0.015)$ were the associated factors of diabetes-related distress, which explained 9.5 percent of the variance. In addition, sleep time $(\beta=-0.379, \mathrm{P}=0.044)$ and GSES $(\beta=-0.153, \quad \mathrm{P}=0.003)$ were the related factors of $\mathrm{EB}$, which explained 28.4 percent of the variance. Physical activity $(\beta=-0.185, \mathrm{P}=0.004)$, treatment regimen with oral medication 
plus insulin the highest $(\beta=0.152, \mathrm{P}=0.018)$, and having meal plan developed by HCPs and the patient $(\beta=-0.169, \mathrm{P}=0.009)$ were relevant to $\mathrm{RD}$, which explained 10.9 percent of the variance. And treatment regimen $(\beta=0.471, \mathrm{P}=0.034)$ was related to $\mathrm{PD}$ and explained 1.5 percent of the variance simultaneously. Meantime, physical activity $(\beta=-0.134, \quad \mathrm{P}=0.010)$, treatment regimen with oral medication plus insulin the highest $(\beta=0.103, \mathrm{P}=0.049)$ were associated factors of ID and explained 2.3 percent of the variance.(See Table 4)

Table 4

Stepwise multiple linear regression for DDS, EB, PD, RD, ID and related factors $(\mathrm{n}=363)$

\begin{tabular}{|c|c|c|c|c|c|}
\hline \multirow[t]{2}{*}{$\begin{array}{l}\text { Dependent } \\
\text { variables }\end{array}$} & \multirow[t]{2}{*}{ Model } & $\begin{array}{l}\text { Unstandardized } \\
\text { coefficients }\end{array}$ & $\begin{array}{l}\text { Standardized } \\
\text { coefficients }\end{array}$ & \multirow[t]{2}{*}{$\mathrm{t}$} & \multirow[t]{2}{*}{$\mathrm{p}$} \\
\hline & & B & $\beta$ & & \\
\hline \multirow[t]{3}{*}{ DDS } & Constant & 44.175 & - & 7.326 & 0.000 \\
\hline & Sleep time & -1.608 & -0.190 & -2.212 & 0.029 \\
\hline & GSES & -1.838 & -0.128 & -2.450 & 0.015 \\
\hline \multirow[t]{3}{*}{ EB } & Constant & 29.834 & - & 4.433 & 0.000 \\
\hline & Sleep time & -1.273 & -0.379 & -2.136 & 0.044 \\
\hline & GSES & -0.893 & -0.153 & -2.944 & 0.003 \\
\hline \multirow[t]{2}{*}{$\mathrm{PD}$} & Constant & 4.855 & - & 0.019 & $<0.01$ \\
\hline & Treatment regimens & 0.471 & 0.137 & 2.137 & 0.034 \\
\hline \multirow[t]{6}{*}{$\mathrm{RD}$} & Constant & 14.713 & - & 7.838 & 0.000 \\
\hline & Physical activity & -2.150 & -0.185 & -2.933 & 0.004 \\
\hline & Treatment regimens & 0.793 & 0.152 & 2.380 & 0.018 \\
\hline & Whether meal plan had & -1.965 & -0.169 & -2.630 & 0.009 \\
\hline & been developed by & & & & \\
\hline & $\mathrm{HCP}$ and the patient & & & & \\
\hline \multirow[t]{2}{*}{ ID } & Constant & 1.541 & - & 12.925 & $<0.01$ \\
\hline & Physical activity & -0.250 & -0.134 & -2.582 & 0.010 \\
\hline
\end{tabular}




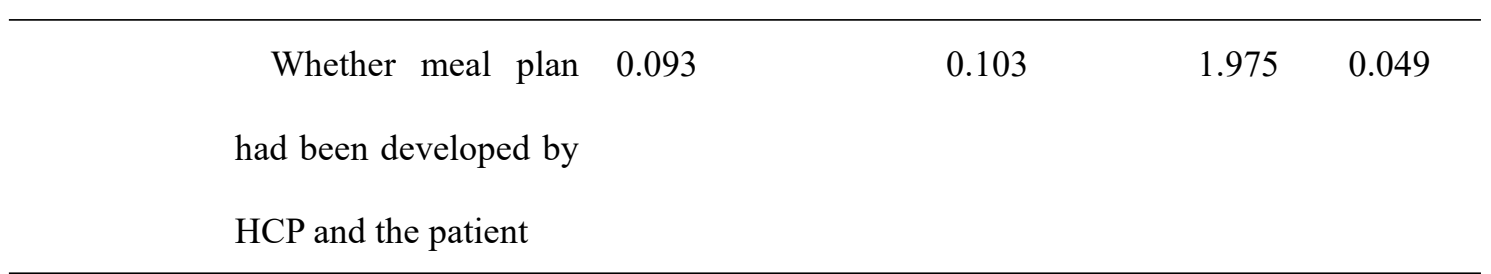

DDS, Diabetes Distress Scale; EB, emotional burden subscale; PD, physician-related distress subscale; $\mathrm{RD}$, regimen-related distress subscale; ID, diabetes-related interpersonal distress subscale; GSES, General Self-Efficacy Scale; HCP, health care professional.

\subsection{The relationship between diabetes-related distress and total sleep time}

We performed quadratic regression for DDS and total sleep time, and we took $\mathrm{B}_{1}=-1.642$ and $\mathrm{B}_{2}=0.095$ into the formula $\beta_{1}+\beta_{2} * X=0$, and finally obtained $X=8.6$. Diabetes-related distress and sleep time was negatively correlated $(\mathrm{P}=0.008)$. When total sleep time was $8.6 \mathrm{~h}$, diabetes-related distress was the lowest. There was no statistical significance between EB and sleep time ${ }^{2}$. (See Table 5)

Table 5

Quadratic regression for DDS and total sleep time $(n=363)$

\begin{tabular}{|c|c|c|c|c|c|}
\hline \multirow[t]{2}{*}{$\begin{array}{l}\text { Dependent } \\
\text { variables }\end{array}$} & \multirow[t]{2}{*}{ Model } & $\begin{array}{l}\text { Unstandardized } \\
\text { coefficients }\end{array}$ & $\begin{array}{l}\text { Standardized } \\
\text { coefficients }\end{array}$ & \multirow[t]{2}{*}{$\mathrm{t}$} & \multirow[t]{2}{*}{$\mathrm{p}$} \\
\hline & & B & $\beta$ & & \\
\hline \multirow[t]{3}{*}{ DDS } & Constant & 34.134 & & 5.826 & $<0.001$ \\
\hline & Sleep time & -1.642 & -0.200 & -2.687 & 0.008 \\
\hline & Sleep time ${ }^{2}$ & 0.095 & 0.189 & 2.524 & 0.012 \\
\hline \multirow[t]{3}{*}{ EB } & Constant & 11.443 & & 4.790 & $<0.001$ \\
\hline & Sleep time & -0.696 & -0.212 & -2.792 & 0.006 \\
\hline & Sleep time ${ }^{2}$ & 0.024 & 0.121 & 1.593 & 0.113 \\
\hline
\end{tabular}

DDS, Diabetes Distress Scale;EB, emotional burden subscale; Sleep time ${ }^{2}=$ total sleep time $\times$ total sleep time.

\section{Discussion}

In the present study, the incidence of diabetes-related distress among Chinese patients was 
high. We found that the unmarried, widowed or divorced, patients using complicated therapy (oral plus insulin), and inactive people got higher diabetes-related distress. Total sleep time and GSES were the associated factors of diabetes-related distress. These indicate that clinicians should pay attention to patients' sleep and take interventions if necessary, and diabetes educator should take strategies to improve patients' self efficacy.

The prevalence of moderate or higher diabetes-related distress was as high as $42.15 \%$, consistent with that (44.6\%) reported by Nicolucci et al. (2013). Therefore, in clinical practice, in addition to depression, HCPs should also pay close attention to diabetes-related distress. The data indicated that being married was related to higher levels of diabetes-related satisfaction and less diabetes-related distress. This finding was in agreement with a study by Trief et al. (2001), which demonstrated that marital status did relate to an individual's adaptation to diabetes. This can be explained as when a patient makes a hospital visit; he or she will be accompanied by his/her spouse. In daily life, his or her spouse may remind the patient on healthy eating, and do exercise with the patient. Therefore, he or she experiences less diabetes-related emotional distress than those without spouses support.

The total score of DDS was not significantly related to patients' gender, age, educational level, diabetes duration, diabetes education, physical activity, whether the meal plan was developed by HCP and the patient, and complications. These findings were similar to those demonstrated by Polonsky WH et al. (2005) and Ting RZ et al. (2011). We did not found that diabetes-related distress was associated with $\mathrm{HbA1c}$, but in a Japanese study diabetes-related distress was associated with poor glycemic control (Hayashino et al., 2012). The results of the two studies may have varied because of the difference of subjects. In our study, we only included patients with type 2 diabetes mellitus, both type 1 and type 2 patients participated in the Japanese study. On the other hand, their sample size is 10 times larger than ours.

To our knowledge, this was the first study to examine the associated factors of diabetes-related distress in Chinese patients with type 2 diabetes mellitus. We found that patients using insulin therapy had a higher level of diabetes-related distress than those with oral hypoglycemic agents only or on lifestyle change only, which was partially consistent with Delahanty et al. (2007) and Makine et al. (2009). Several reasons might explain this 
phenomenon. First, compared with oral medication, insulin injection is relatively complicated (Demirci et al., 2010) and is difficult for the elderly (Wong et al., 2011). Second, patients treated with insulin have been found to be in poor glycemic control (Holman et al., 2009) and have longer duration diabetes with complications. Moreover, the cost of insulin is higher (Polinski et al., 2013). We also found that insulin plus oral medication users reported the highest DDS. In order to deal with the complicated medication regimens, patients needed to spend more energy and had higher medicine expenses, which could cause greater distress. Therefore, for patients treated with insulin, HCPs should focus on assessing patients' psychological problems, such as diabetes-related distress, depression and anxiety.

Furthermore, our study indicated that $19.0 \%$ individuals with type 2 diabetes mellitus had total sleep time of 6.5 hours, and less sleep time was related to higher diabetes-related distress. Previous study demonstrated that sleep time of 5 hours or less was associated with an increased prevalence of diabetes mellitus and an impaired glucose tolerance test (Najafian et al., 2013). Poor sleep might bring much more serious insulin resistance and could be the reason for poor glycemic control (Reutrakul et al., 2015). In addition, sleep insufficiency together with psychological distress had an interactive influence on the living quality of type 2 diabetes mellitus patients. A good night's sleep should be seen as a critical health component in the prevention and treatment of type 2 diabetes mellitus. Thus, type 2 diabetes mellitus patients should ensure they get 8.6 hours of sleep per day and understand why that is so very important.

Self-efficacy is a positive characteristic that has been frequently studied. Higher self-efficacy was a significant factor related to lower diabetes-related distress (Wardian and Sun, 2014). In this study in the stepwise multiple linear regression, self-efficacy was negatively associated with diabetes-related distress. Higher scores on the GSES indicated lower diabetes-related distress in patients with type 2 diabetes mellitus. Our finding was in agreement with the results of two American studies (Wardian and Sun, 2014; Hessler et al., 2011).

The study had some limitations. First, the study population was from a single hospital in Nanjing, Jiangsu Province, China, which may limit generalization. Second, the design of 
cross-section study may have led to a selection bias, like berkson bias. Compared with outpatients, inpatients were in serious conditions. At the same time, environmental change also made a difference in sleep. Third, there was memory bias in the study, especially when we asked some questions about sleep time, hypoglycemia, complications and so on, patients had to recall what had happened in the past, which was not $100 \%$ correct. In addition, the diabetes duration of patients was at least 3 months. This could affect the real evaluation of diabetes-related distress being the first period of the disease (almost the first year after the diagnosis) peculiar in term of psychological status compared to a longer disease duration. Finally, Rasch analysis was not conducted on the DDS and GSES which is a shortcoming of the study. Because previous work has shown the DDS to have suboptimal psychometric properties especially its subscales which may bring into question the results in the current study (Fenwick et al., 2016) .

In conclusion, the prevalence of diabetes-related distress among patients with type 2 diabetes mellitus was high in China. Lack of sleep and low self-efficacy were factors associated of diabetes-related distress. Interventions to improve sleep are needed. Both qualitative studies to understand why patients are not getting enough sleep and longitudinal studies are needed. Meanwhile, complicated treatment regimens with insulin, especially oral medication plus insulin, was associated with psychological distress. Findings of this study suggest that for patients treated with insulin, HCPs should help them to deal with injection-related concerns and perceived lifestyle adaptations.

\section{Funding}

This study was not funded by any one.

\section{Conflict of interest}

No potential conflicts of interest relevant to this article were reported.

\section{Acknowledgment}

The authors thank the patients and researchers at Affiliated Hospital of Integrated 
Traditional Chinese and Western Medicine, Nanjing University of Chinese Medicine for participating in this study. The authors thank Xiaodan Yuan for her assistance with data collection.

\section{References}

Aikens J.E., 2012. Prospective Associations Between Emotional Distress and Poor Outcomes in Type 2 Diabetes. Diabetes Care 35(12): 2472-2478.

Ali M.K., Bullard K.M., Saaddine J.B., Cowie C.C., Imperatore G., Gregg E.W., 2013. Achievement of goals in U.S. diabetes care, 1999-2010. N Engl J Med 368(17):1613-1624. American Diabetes Association, 2016. Standards of medical care in diabetes-2016. Diabetes Care 39 (Suppl. 1): S13-S22.

Brieler J.A., Lustman P.J., Scherrer J.F., Salas J., Schneider F.D., 2016. Antidepressant medication use and glycaemic control in co-morbid type 2 diabetes and depression. Fam Pract 33(1), 30-36.

Chew BH, Vos R, Mohd-Sidik S, Rutten GE, 2016. Diabetes-Related Distress, Depression and Distress-Depression among Adults with Type 2 Diabetes Mellitus in Malaysia. PLoS One. 11(3): e0152095.

Delahanty L.M., Grant R.W., Wittenberg E., Bosch J.L., Wexler D.J., Cagliero E., Meigs J.B., 2007. Association of diabetes-related emotional distress with diabetes treatment in primary care patients with Type 2 diabetes. Diabet Med 24(1), 48-54.

Demirci H., Cinar Y., 2010. The predictive role of $\mathrm{HbA}_{1}(\mathrm{c})$ and previous medications in initiation of insulin treatment. Dan Med Bull 57(11): A4214.

Fenwick E.K., Rees G., Holmes-Truscott E., Browne J.L., Pouwer F., Speight J., 2016. What is the best measure for assessing diabetes distress? A comparison of the Problem Areas in Diabetes and Diabetes Distress Scale: results from Diabetes MILES-Australia. J Health Psychol pii: 1359105316642006.

Fisher L., Hessler D.M., Polonsky W.H., Mullan J., 2012. When is diabetes distress clinically meaningful?: establishing cut points for the Diabetes Distress Scale. Diabetes Care 35, $259-264$. 
Fisher L., Mullan J.T., Arean P., Glasgow R.E., Hessler D., Masharani U., 2010. Diabetes distress but not clinical depression or depressive symptoms is associated with glycemic control in both cross-sectional and longitudinal analyses. Diabetes Care $33(1): 23-28$.

Graue M., Haugstvedt A., Wentzel-Larsen T., Iversen M.M., Karlsen B., Rokne B., 2012. Diabetes-related emotional distress in adults: reliability and validity of the Norwegian versions of the Problem Area in Diabetes Scale (PAID) and the Diabetes Distress Scale (DDS). Int J Nurs Stud 49, 174-182.

Hayashino Y., Okamura S., Matsunaga S., Tsujii S., Ishii H., Tenri Cohort Study Group, 2012. The association between problem areas in diabetes scale scores and glycemic control is modified by types of diabetes therapy: diabetes distress and care registry in Tenri (DDCRT 2). Diabetes Res Clin Pract 97(3):405-410.

Hessler D.M., Fisher L., Mullan J.T., Glasgow R.E., Masharani U., 2011. Patient age: A neglected factor when considering disease management in adults with type 2 diabetes. Patient Educ Couns 85(2), 154-159.

Holman R.R., Farmer A.J., Davies M.J., Levy J.C., Darbyshire J.L., Keenan J.F., Paul S.K.; 4-T Study Group, 2009. Three-year efficacy of complex insulin regimens in type 2 diabetes. N Engl J Med 361(18): 1736-1747.

Karlsen B., Bru E., 2014. The relationship between diabetes-related distress and clinical variables and perceived support among adults with type 2 diabetes A prospective study. International Journal of Nursing Studies 51, 438-447.

Kawada T., 2016. Anxiety and Depression in Female Patients With Type 2 Diabetes. Am J Public Health 106(2), e1.

Kong L., Cai Y., Mei G., 2013. Psychological status and diabetes-related distress of Chinese type 1 diabetes patients in Jiangsu province, China. J Biomed Res 27(5), 380-385.

Luyster F.S., Dunbar-jacob J., 2011. Sleep quality and quality of life in adults with type 2 diabetes. Diabetes Educ 37, 347-355.

Makine C., Karşidağ C., Kadioğlu P., Ilkova H., Karşidağ K., Skovlund S.E., Snoek F.J., Pouwer F., 2009. Symptoms of depression and diabetes-specific emotional distress are 
associated with a negative appraisal of insulin therapy in insulin-naïve patients with Type 2 diabetes mellitus. A study from the European Depression in Diabetes[EDID] Research Consortium. Diabet Med 26(1), 28-33.

Munhoz T.N., Nunes B.P., Wehrmeister F.C., Santos I.S., Matijasevich A., 2015. A nationwide population-based study of depression in Brazil. J Affect Disord 192, 226-233.

Najafian J., Mohamadifard N., Siadat Z.D., Sadri G., Rahmati M.R., 2013. Association between sleep duration and diabetes mellitus: Isfahan Healthy Heart Program. Nigerian Journal of Clinical Practice 16 (1), 59-62.

Nicolucci A., Kovacs Burns K., Holt R.I.G., Comaschi M., Hermanns N., Ishii H., Kokoszka A., Pouwer F., Skovlund S.E., Stuckey H., Tarkun I., Vallis M., Wens J., Peyrot M., 2013. Diabetes Attitudes, Wishes and Needs second study (DAWN2 ${ }^{\mathrm{TM}}$ ): Cross-national benchmarking of diabetes-related psychosocial outcomes for people with diabetes. Diabetic Medicine 30 (7), 767-777.

Ogbera A., Adeyemi-Doro A., 2011. Emotional distress is associated with poor self care in type 2 diabetes mellitus. Journal of diabetes 3, 348-352.

Park C.Y., Brown J.B., 2015. Unadjusted and adjusted prevalence of diagnosed depression in type 2 diabetes. Osong Public Health Res Perspect 6(4), 224-232.

Polinski J.M., Smith B.F., Curtis B.H., Seeger J.D., Choudhry N.K., Connolly J.G., Shrank W.H., 2013. Barriers to insulin progression among patients with type 2 diabetes: a systematic review. Diabetes Edu 39(1): 53-65.

Polonsky W.H., Fisher L., Earles J., Dudl R.J., Lees J., Mullan J., Jackson R.A., 2005. Assessing psychosocial distress in diabetes: development of the diabetes scale. Diabetes Care $28,626-631$.

Reutrakul S., Siwasaranond N., Nimitphong H., Saetung S., Chirakalwasan N., Ongphiphadhanakul B., Thakkinstian A., Hood M.M., Crowley S.J., 2015. Relationships among sleep timing, sleep duration and glycemic control in Type 2 diabetes in Thailand. Chronobiol Int 32(10): 1469-1476.

Schwarzer R., Born A., 1997. Optimistic self-beliefs: Assessment of general perceived self-efficacy in Thirteen cultures. World Psychology 3,177-190. 
Stanković Z., Jasović-Gasić M., Lecić-Tosevski D., 2013. Psychological problems in patients with type 2 diabetes-clinical considerations. Vojnosanit Pregl 70(12), 1138-1144.

Ting R.Z., 2011. Diabetes-related distress and physical and psychological health in chinese type 2 diabetic patients. Diabetes Care 34(5), 1094-1096.

Trief P.M., Himes C.L., Orendorff R., Weinstock R.S., 2001. The Marital Relationship and Psychosocial Adaptation and Glycemic Control of Individuals With Diabetes. Diabetes Care 24, 1384-1389.

Wardian J., Sun F., 2014. Factors Associated with Diabetes-related Distress: Implications for Diabetes Self-Management. Soc Work Health Care 53(4), 364-381.

Wong S., Lee J., Ko Y., Chong M.F., Lam C.K., Tang W.E., 2011. Perceptions of insulin therapy amongst Asian patients with diabetes in Singapore. Diabet Med 28: 206-211.

Yang Q., Liu X.Q., 2010. Reliability and validity of the diabetes distress scale. Journal of Nursing (China) 17, 8-10. Chinese

Zhang J.X., Schwarzer R., 1995. Measuring optimistic self-beliefs: A Chinese adaptation of the General Self-efficacy Scale. Psychologia 38, 174-181.

Zhang J., Xu C.P., Wu H.X., Xue X.J., Xu Z.J., Li Y., Gao Q., Liu Q.Z., 2013. Comparative study of the influence of diabetes distress and depression on treatment adherence in Chinese patients with type 2 diabetes: a crosssectional survey in the People's Republic of China. Neuropsychiatric Disease and Treatment 9, 1289-1294.

Zhang P., Lou P., Chang G., Chen P., Zhang L., Li T., Qiao C., 2016. Combined effects of sleep quality and depression on quality of life in patients with type 2 diabetes. BMC Fam Pract 17: 40.

Zhao J., Li X.L., Han K., Tao Z.Q., Wu Z.M., 2016. Biological interaction between sleep quality and depression in type 2 diabetes. Eur Rev Med Pharmacol Sci 20 (14): 3087-3091. 